\title{
Prise en compte de la variabilité individuelle dans la modélisation de la réponse des porcs en croissance aux apports alimentaires
}

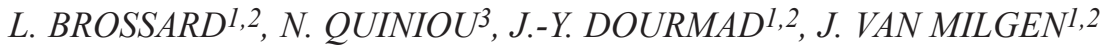 \\ ${ }^{1}$ INRA, UMR1348 Physiologie, Environnement et Génétique pour l'Animal et les Systèmes d'Elevage, \\ F-35590 Saint-Gilles, France \\ 2 Agrocampus Ouest, UMR Physiologie, Environnement et Génétique pour l'Animal et les Systèmes d'Elevage, \\ F-35000 Rennes, France \\ ${ }^{3}$ IFIP- Institut du Porc, Pôle Techniques d'Elevage, BP 35104, F-35651 Le Rheu, cedex, France \\ Courriel : ludovic.brossard@rennes.inra.fr
}

La maîtrise des coûts alimentaires et des impacts environnementaux de l'élevage des porcs en croissance passe par une meilleure connaissance de la réponse des animaux aux apports nutritionnels, et donc de leurs besoins, notamment en acides aminés, mais également de la variabilité de ces derniers. La question est alors : comment évaluer cette variabilité et l'intégrer dans les modèles de croissance pour améliorer l'évaluation multicritère des stratégies alimentaires ? 1

En nutrition des porcs à l'engraissement, l'optimisation de l'efficacité alimentaire et de la qualité de carcasse sont deux objectifs recherchés depuis longtemps par les concepteurs d'aliment afin d'améliorer le retour économique de cette production. Plus récemment, d'autres critères sont apparus tels que la réduction de l'impact environnemental, l'adaptation de la qualité des produits aux demandes des consommateurs (par exemple en termes de nature des acides gras dans la viande) ou l'amélioration du bien-être des animaux (satisfaction des besoins ou de l'appétit). Pour que les apports correspondent au mieux aux besoins, de façon à minimiser les rejets résultant du déséquilibre de certains nutriments, la formulation des régimes implique une connaissance précise des lois de réponse aux nutriments, et donc des besoins nutritionnels des animaux et de leur évolution au cours du temps. Les performances de croissance et d'ingestion des porcs à l'engraissement dépendent de facteurs liés à l'animal (type génétique, type sexuel, stade de croissance, statut social au sein du groupe) et aux conditions d'élevage (qualité et quantité des aliments alloués, taille du groupe, surface disponible, température ambiante, qualité de l'air...) (Quiniou et
Massiot 2008). Ceci se traduit au sein d'un élevage par des performances variables entre groupes mais aussi entre animaux au sein d'un même groupe, même si leur type génétique et les conditions de logement et de statut sanitaire sont identiques (Quiniou et al 2012). Cette variabilité des performances s'accompagne d'une variabilité des besoins nutritionnels, par exemple en acides aminés (Noblet et Quiniou 1999, Wecke et Liebert 2009).

Historiquement, les besoins nutritionnels ont été déterminés par des lois de réponse empiriques décrivant la croissance en fonction des apports nutritionnels et publiés sous forme de tables. Ces lois de réponse étaient calculées à partir de la réponse moyenne des animaux, le plus souvent sans accorder d'intérêt aux variances observées autour de la moyenne. Cette démarche pose le problème de l'extrapolation des résultats dans d'autres conditions que celles des essais. Une estimation se basant sur une représentation des mécanismes physiologiques impliqués a donc été proposée. Elle vise à prendre en compte l'influence des facteurs internes ou externes et de leurs interactions sur les besoins tout en intégrant les lois de réponse connues, ainsi que proposé dans différents modèles mathématiques mécanistes tels que celui du NRC (1998) ou de l'INRA (van Milgen et al 2008). Ces modèles présentent cependant certaines difficultés d'application, les animaux pouvant en pratique présenter des trajectoires de croissance et d'ingestion différentes de celles attendues. Ces écarts peuvent ainsi résulter du fait que les animaux sont élevés en groupes hétérogènes (génétique, sexe, facteurs d'élevage), hétérogénéité difficile à quantifier, ou dans des conditions d'élevage variables entre sites de production, notamment en termes de statut sanitaire (Pomar et al 2009a).

D'autres modèles mathématiques ont été développés pour simuler la croissance ou estimer les besoins nutritionnels, intégrant une dimension dynamique par la représentation de l'évolution temporelle des performances et des besoins. Ces modèles se basent sur un individu unique, censé être représentatif d'une population, à partir d'informations collectées sur des populations d'animaux similaires (van Milgen et al 2008, Pomar et al 2009a). Or, la réponse d'un individu aux apports nutritionnels et celle de la population qu'il est supposé

\footnotetext{
${ }^{1}$ Cette synthèse fait suite à une réunion de la Commission Porcine de l'INRA consacrée au thème de «la variabilité en élevage porcin». Les auteurs sont membres de l’Unité Mixte Technologique Ingénierie des systèmes de production porcine.
} 
représenter peuvent différer (Pomar et al 2003, Wellock et al 2004). Dans ce contexte, l'objectif de cet article est, d'une part, de montrer comment la variabilité des besoins nutritionnels des porcs peut être évaluée et, d'autre part, de démontrer l'intérêt de l'intégration de cette variabilité individuelle dans les modèles de simulation.

\section{1 / Détermination expéri- mentale de la variabilité des besoins en acides aminés}

Compte tenu de l'évolution des performances induite par la sélection génétique et de la volonté d'améliorer l'adéquation entre les apports alimentaires et les besoins des animaux, la détermination des besoins nutritionnels du porc en croissance reste encore un sujet d'actualité. Différentes études se sont intéressées à la détermination des besoins en différents acides aminés chez le porc en croissance ou le porcelet (Warnants et al 2003, Heger et al 2008 et 2009, Moehn et al 2008, Barea et al 2009). Si les besoins moyens dépendent de nombreux facteurs intrinsèques, des conditions d'élevage et des caractéristiques alimentaires (Noblet et Quiniou 1999, Wecke et Liebert 2009), il existe également une variabilité du besoin entre animaux (Bertolo et al 2005). La détermination expérimentale de ces besoins repose classiquement sur une approche empirique basée sur la mesure de lois de réponse en termes de gain de poids, d'efficacité alimentaire ou de bilan azoté à un apport croissant de l'acide aminé étudié. Selon le critère et l'étude, la réponse à l'apport en acide aminé est mesurée individuellement ou collectivement sur des groupes d'animaux. Barea et al (2009) mesurent ainsi individuellement l'évolution du poids et de la consommation d'aliment. Warnants et al (2003) mesurent la consommation alimentaire à l'échelle de groupes de cinq animaux et le poids individuellement sur chacun des individus de ces groupes, le groupe étant en définitive l'unité expérimentale considérée dans l'analyse statistique des résultats. Les lois de réponse sont calculées à partir de la réponse moyenne obtenue à partir de l'ensemble des individus, le plus souvent sans rapporter la variabilité observée autour de ces moyennes. Par ailleurs, dans ce type d'étude, chaque individu ne reçoit au cours de l'expérience qu'un niveau d'apport en acide aminé. En effet, les méthodes basées sur les mesures de performances nécessitent de longues périodes de mesures ou d'adaptation au régime pour obtenir des résultats valides. La durée de l'étude de la réponse d'un même animal à toutes les teneurs souhaitées en acide aminé
Figure 1. Fréquence de distribution des besoins prévus en lysine digestible standardisée chez des porcs en croissance d'après la moyenne et l'Ecart-Type (ET) obtenus expérimentalement à $24,5 \mathrm{~kg}$ de poids moyen (adapté de Bertolo et al 2005). Les besoins mesurés pour chaque individu sont indiqués par un point rouge.

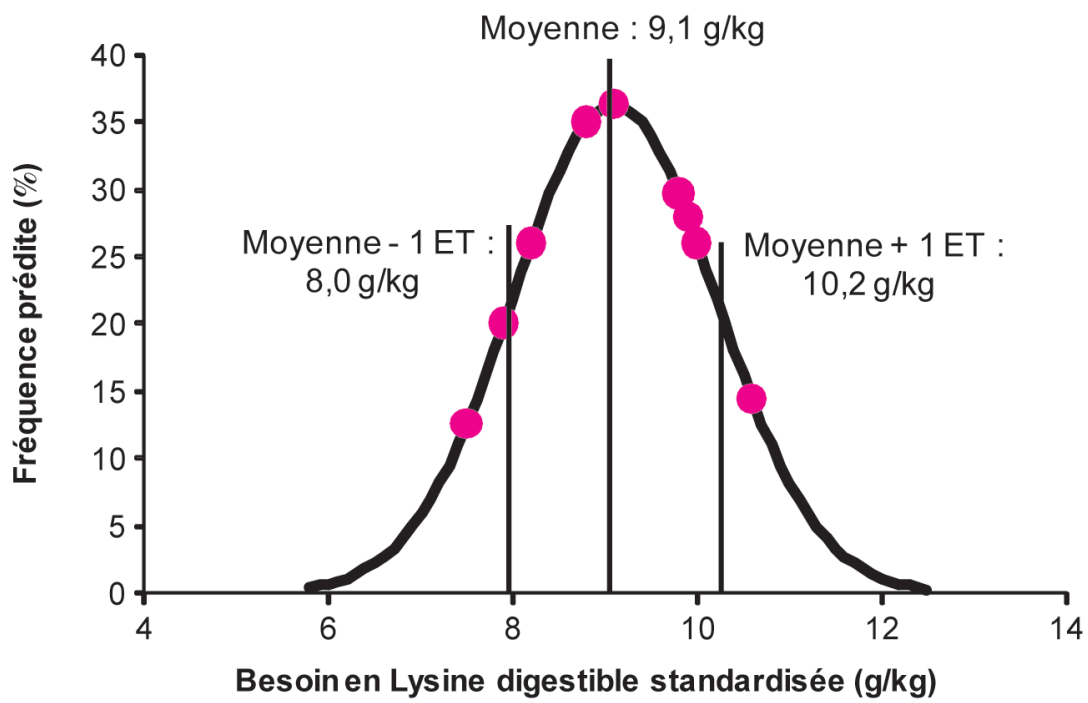

serait donc trop importante (plusieurs mois), même dans le cadre d'un essai en carré latin. En effet, elle impliquerait une évolution du stade physiologique de l'animal et de ses besoins. Une loi de réponse individuelle à des apports variables de l'acide aminé ne peut donc pas être établie par ce type de méthode.

Pour l'obtention de valeurs individuelles de besoin, Heger et al (2007, 2008, 2009) ont utilisé une méthode de bilans azotés consécutifs de 24 heures chacun, en réponse à de petites augmentations de l'acide aminé limitant dans le régime. Après 4 jours d'adaptation au régime le plus carencé, chaque animal étudié reçoit consécutivement sur 15 jours, 15 niveaux d'apport de l'acide aminé étudié augmentant graduellement de 50 à $140 \%$ du besoin estimé. Les régimes semi purifiés utilisés sont à base de caséine et d'acides aminés cristallins. Pour effectuer les bilans, l'urine est collectée le lendemain de l'application d'un régime, avant l'apport du régime suivant. Pour un régime donné, les fèces sont collectées 4 jours après l'ingestion du régime pour tenir compte du temps moyen de transit des digesta. L'ajustement d'un modèle quadratique plateau ou linéaire-plateau à la réponse de chaque individu permet d'obtenir le besoin en l'acide aminé étudié pour chaque porc et sa variabilité entre les animaux étudiés. Ces auteurs évaluent ainsi expérimentalement sur neuf femelles croisées Large White $x$ (Large White $x$ Landrace) le Coefficient de Variation (CV) du besoin en acide aminé (en valeur digestible standardisée) à $3 \%$ à $45 \mathrm{~kg}$ de Poids Vif (PV) pour la thréonine (Heger et al 2007), à $11 \%$ à $50 \mathrm{~kg}$ de PV pour la méthionine (Heger et al 2008) et à $10 \%$ à $47 \mathrm{~kg}$ de PV pour la lysine (Heger et al 2009).
La méthode d'oxydation d'un acide aminé indicateur, initialement développée chez le porcelet (Kim et al 1983), a été utilisée chez le porc en croissance (Bertolo et al 2005, Moehn et al 2008) et permet l'obtention de valeurs individuelles du besoin. Son concept suppose que le déficit même faible en un acide aminé essentiel pour la synthèse protéique entraîne l'oxydation des autres acides aminés essentiels, dont l'acide aminé indicateur, apporté lui en quantité constante. L'ingestion croissante de l'acide aminé limitant induit donc une diminution de l'oxydation de l'acide aminé indicateur (le plus souvent la phénylalanine), indiquant son assimilation accrue dans les protéines. Cette méthode ne nécessite qu'une courte période d'adaptation. Moehn et al (2004) ont ainsi démontré que 2 jours étaient suffisants pour obtenir une réponse oxydative stable quel que soit le niveau de carence ou d'excès de l'acide aminé étudié ou le niveau de turn-over protéique. La réponse d'un même animal à un nombre suffisant de niveaux d'apport de l'acide aminé testé pour déterminer une loi de réponse peut être obtenue en 2 à 3 semaines, donc sans changement majeur du statut physiologique de l'animal sur la période. En appliquant cette technique à neuf mâles castrés issus d'un croisement commercial et recevant des niveaux d'apports en lysine variant entre 56 et $145 \%$ des recommandations du NRC (1998), Bertolo et al (2005) évaluent le CV du besoin moyen en lysine digestible standardisée à $11,6 \%$ à $24 \mathrm{~kg}$ de PV. Ces auteurs observent également que les valeurs individuelles de besoin en lysine déterminées dans cette étude sont distribuées normalement autour de la moyenne (figure 1). 
Les techniques de bilans azotés consécutifs et d'oxydation d'un acide aminé indicateur permettent la maîtrise des conditions expérimentales, de l'ingestion et du poids des animaux et donc de circonscrire les sources de variabilité à celles liées intrinsèquement à l'animal. Ainsi, en répétant les mesures sur plusieurs animaux, la variabilité au sein d'une population peut être estimée. Cependant, ces deux techniques sont relativement compliquées et coûteuses. La technique d'oxydation de l'acide aminé indicateur nécessite par exemple des animaux munis de cathéters, l'utilisation d'éléments marqués $\left({ }^{13} \mathrm{C}\right.$ ou ${ }^{14} \mathrm{C}$ phénylalanine) et une chambre respiratoire, le niveau d'oxydation étant mesuré par la quantité expirée de $\mathrm{CO}_{2}$ marqué. Le nombre d'animaux étudiés est donc limité et la variabilité observée ne peut refléter que partiellement celle de populations plus grandes. De plus, les animaux étudiés sont logés individuellement. Les interactions au sein d'un groupe influençant l'expression de la variabilité intrinsèque, la représentativité de ces animaux, et donc de la variabilité observée, par rapport à des animaux logés en groupe peut poser question. Ces contraintes impliquent également qu'il soit difficile d'étudier expérimentalement la dynamique des besoins des animaux au cours de l'ensemble de la croissance en répétant les mesures sur les mêmes individus. Cette dynamique et sa variabilité sont donc peu étudiées expérimentalement alors que ces connaissances seraient nécessaires pour améliorer l'ajustement des apports nutritionnels aux besoins des animaux.

\section{2 / Intégration de la variabi- lité dans la modélisation de la croissance chez le porc}

\section{1 / Impact de la variabilité individuelle sur les réponses des modèles de croissance}

La modélisation mathématique s'est développée en nutrition porcine afin d'intégrer les connaissances disponibles et représenter l'incidence des principaux facteurs impliqués dans la réponse animale (Pomar 2005). Ainsi, plusieurs modèles de la croissance du porc ont été développés (Whittemore et Fawcett 1976, Moughan et al 1987, Knap 2000a, van Milgen et al 2008). Ces modèles peuvent être utilisés pour simuler de façon dynamique les performances d'un individu selon le contexte nutritionnel ou les conditions d'élevage, et pour estimer les besoins nutritionnels des animaux. Cependant, ils se limitent à la description d'un individu moyen et leur paramétrage repose sur l'acquisition d'informations à partir de la population que cet individu moyen est censé représenter (Pomar et al 2009a). Les paramètres qui caractérisent l'individu moyen ne varient donc pas pendant la simulation ni entre simulations, ce qui correspond à un processus de modélisation déterministe.

Différents éléments peuvent être avancés pour ne plus considérer uniquement l'individu moyen mais également la variabilité animale dans les modèles, qui deviennent alors stochastiques, c'est-àdire qu'une variabilité liée aux individus est associée à certains paramètres du modèle. Dans l'optique de simuler et comparer différents systèmes de production ou stratégies alimentaires, Knap (1995) expose cinq arguments en faveur de l'intégration de la variabilité : 1/ la profitabilité des systèmes de production peut être affectée en grande partie par l'ampleur de la variabilité des performances zootechniques ; 2/ le passage d'un système de production à un autre peut avoir des effets mineurs sur les niveaux moyens mais des effets importants sur la variabilité. Par exemple, comparativement à un niveau alimentaire restreint, une distribution ad libitum en fin de croissance peut présenter un impact faible sur la moyenne du Gain Quotidien Moyen (GMQ) des animaux. Elle peut en revanche entraîner une augmentation de la variabilité du GMQ entre animaux, ce qui influencera la distribution des poids de sortie et du Taux de Muscle des Pièces (TMP), et donc le paiement des carcasses. 3/ Une comparaison argumentée des systèmes de production nécessite des tests statistiques et donc la connaissance de la variabilité au sein et entre les systèmes. En effet, une différence entre les moyennes d'un paramètre selon deux situations n'a de sens que si elle est mise en regard de la variation au sein de chaque situation, variation qu'il faut donc connaître. 4/ Les différences entres systèmes peuvent être découvertes plus facilement quand la variabilité est visible, notamment en rendant visible les distributions des critères de réponse étudiés. 5/ Pour étudier les relations entre les différents critères de performances (par exemple la corrélation entre le GMQ et le dépôt de gras selon le niveau d'ingestion), il faut créer de la covariance entre ces critères et donc de la variabilité.

La réponse moyenne d'une population, par exemple à une stratégie alimentaire, est différente en forme et en ampleur lorsqu'elle est calculée à partir des résultats de tous les individus ou à partir de l'individu moyen (Wellock et al 2004). Ceci est illustré par Pomar et al (2003) qui utilisent un modèle de croissance permettant de prévoir l'ingestion, la composition corporelle et les dépôts corporels protéiques et lipi- diques. La composition du régime et le type génétique du porc sont des entrées du modèle. Un porc y est décrit génétiquement par trois paramètres : la masse protéique à maturité $(\mathrm{Pm})$, le niveau intrinsèque d'adiposité caractérisé par le rapport entre masse lipidique et masse protéique à maturité (LPRm), et la vitesse de maturation (constante $B$, ou précocité). Le potentiel de croissance protéique est simulé sous la forme d'une fonction de Gompertz. Le potentiel de croissance lipidique est prédit en fonction de LPRm et Pm. Les besoins journaliers en énergie et acides aminés sont déterminés à partir de la composition corporelle et des potentiels de dépôt protéique et lipidique journaliers chez des porcs nourris à volonté à la neutralité thermique. De plus, comme souvent en modélisation de la croissance du porc, la quantité de protéines déposées dans les tissus en fonction des acides aminés ingérés est déterminée selon une relation linéaire-plateau où le plateau représente le dépôt protéique maximal. Pomar et al (2003) et Pomar (2005) ont utilisé ce modèle pour simuler la croissance de 2500 porcs issus de cinq populations distinctes par leur variance génétique, mais présentant un potentiel génétique moyen de croissance identique, c'est-à-dire des valeurs moyennes identiques pour Pm, LPRm, et un paramètre $\mathrm{B}^{*}\left(\mathrm{~B} \times \mathrm{Pm}^{0,27}\right)$. Comme montré par Ferguson et al (1997), l'utilisation du paramètre $\mathrm{B}^{*}$ permet d'obtenir des corrélations très faibles entre $\mathrm{Pm}$, LPRm et B*, ce qui n'est pas le cas avec le paramètre $B$. Ceci permet de générer des valeurs de façon indépendante pour chaque paramètre à partir de sa moyenne et de son Ecart-Type (ET) sans avoir à connaître les covariances avec les autres paramètres, covariances qui sont difficiles à estimer. Les populations ont été générées aléatoirement afin d'obtenir pour chaque paramètre $0,0,5,1,1,5$ et 2 fois la variance génétique estimée d'une population de référence. La population ayant une variance nulle revient à considérer un animal moyen, unique. $\mathrm{La}$ population avec une variance égale à une fois celle de la population de référence représente une population d'hétérogénéité normale. Les populations avec des variances inférieures ou supérieures à celle de la population de référence correspondent respectivement à des populations peu ou fortement hétérogènes. En supposant que les porcs pèsent tous $50 \mathrm{~kg}$ de $\mathrm{PV}$ au début et que leur consommation est restreinte à $1,9 \mathrm{~kg} / \mathrm{j}$, les performances des populations de porcs ont été simulées sur un jour avec 11 aliments, faisant ainsi varier la quantité de protéine idéale ingérée entre 212 et $290 \mathrm{~g} / \mathrm{j}$.

L'évolution du dépôt protéique en réponse à la quantité de protéine idéale 
Figure 2. Effet de la variabilité entre les individus (0, 0,5, 1, 1,5 et 2 fois l'Ecart-Type de la population de référence) des paramètres du modèle de croissance et de la quantité de protéine idéale ingérée sur le dépôt protéique moyen journalier $(A)$ et le nombre de porcs sous-alimentés (B) pour des populations de porcs de $50 \mathrm{~kg}$ de poids vif (d'après Pomar 2005).

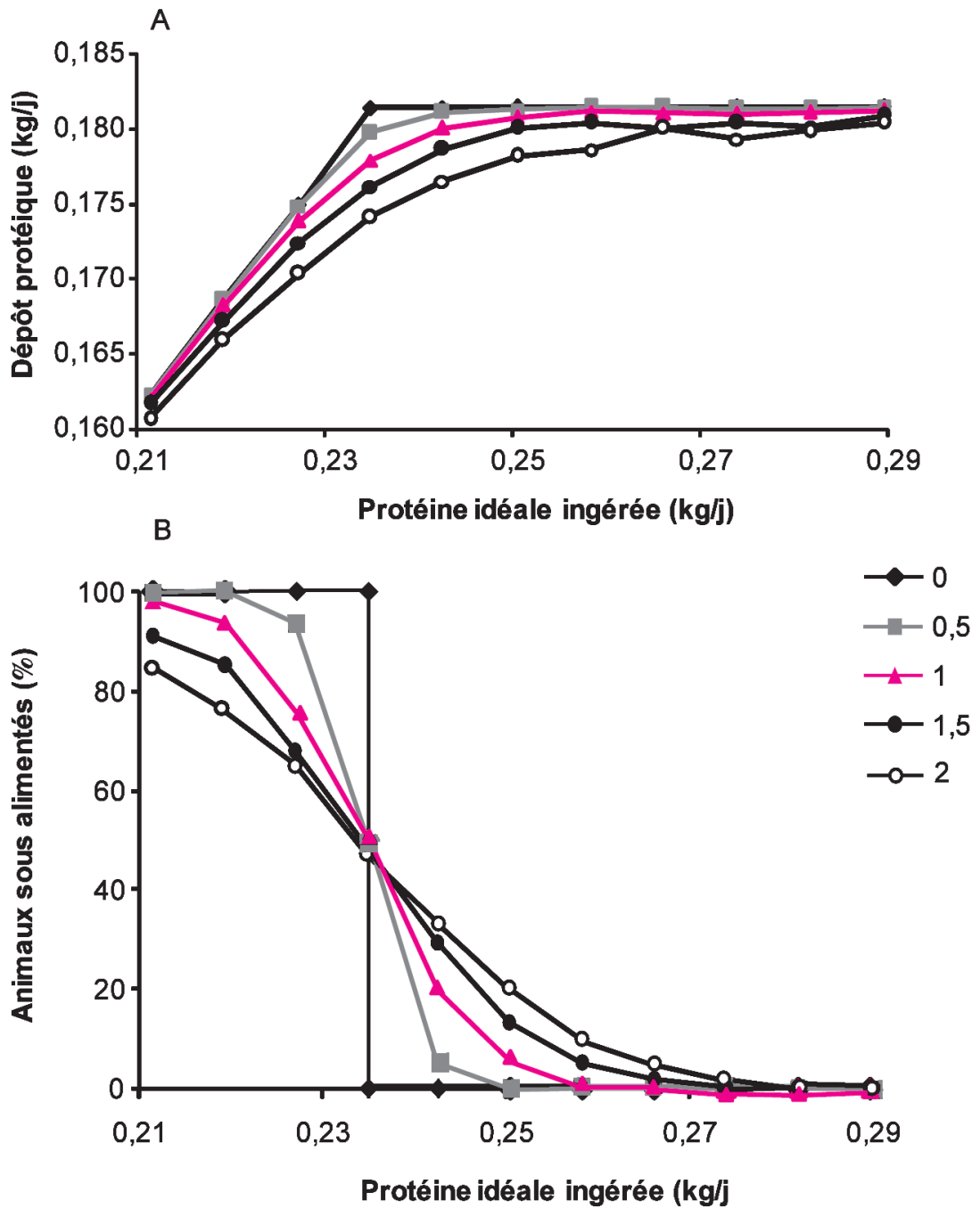

consommée est illustrée par la figure 2A. Pour la population de variance nulle, cette réponse est de type linéaireplateau, en accord avec les hypothèses initiales. La réponse devient de type curvilinéaire-plateau avec une phase curvilinéaire dont l'arrondi et l'amplitude sont de plus en plus marqués quand la variabilité augmente. Le plateau est également atteint pour des valeurs de protéine idéale ingérée qui augmentent avec l'accroissement de la variabilité. Cette relation curvilinéaire-plateau est également observée dans plusieurs études portant sur l'évolution des performances selon l'apport de différents nutriments. Si le besoin nutritionnel est défini comme la quantité de nutriments nécessaire pour maximiser un critère de production (GMQ, efficacité alimentaire...), ces résultats montrent qu'il augmente avec la variabilité de la population. Ainsi, Pomar et al (2003) indiquent des besoins en protéine idéale de 235 , 245 et $251 \mathrm{~g} / \mathrm{j}$ pour maximiser le dépôt bilité. Pour un apport inférieur au besoin en protéine idéale de l'animal moyen, le pourcentage d'animaux sous alimentés diminue d'autant plus que la variabilité augmente. Cette relation est inverse pour des apports supérieurs au besoin de l'animal moyen. Ceci a des conséquences en termes de conduites alimentaires pour optimiser les performances d'un groupe (cf. § 3).

L'exemple présenté ci-dessus repose sur l'intégration d'une seule source de variabilité entre animaux (potentiel de dépôt protéique). Wellock et al (2004) ont étudié l'effet de l'incorporation d'autres sources de variabilité, notamment environnementales, dans le même modèle et en se basant sur les mêmes valeurs de moyenne et d'ET pour les paramètres Pm, LPRm et B* que Pomar et al (2003). La variabilité du poids initial a été introduite en générant des poids initiaux individuels tenant compte notamment des corrélations de ces poids avec Pm, LPRm et B*, un animal à fort potentiel de croissance ayant tendance à présenter un poids initial plus élevé. L'effet de la température ambiante, qui impacte l'ingestion et l'utilisation des nutriments via les mécanismes de thermorégulation, a également été étudié. Enfin, la variabilité de la capacité des animaux à faire face au stress lié à leur environnement social (défini ici par la taille du groupe, la surface par animal dans la case et au nourrisseur et le mélange entre groupes) a été introduite dans le modèle par un paramètre EX. Il exprime l'intensité du facteur à laquelle l'animal devient stressé et l'étendue de la réduction de performance qui en résulte (Wellock et al 2003). Un changement d'une unité du paramètre EX induit une déviation de $1 \%$ de la performance moyenne. La valeur individuelle de EX est calculée en tenant compte notamment d'une corrélation négative avec le poids initial. En faisant varier l'ET soit du poids initial (de 0 à $12 \mathrm{~kg}$ ), soit de EX (de 0 à 2,5) dans des populations dont la croissance était simulée entre 60 et $100 \mathrm{~kg}$ de PV, Wellock et al (2004) n'ont pas observé d'effet sur les valeurs moyennes de GMQ ou de consommation alimentaire. Ils ont par contre observé une augmentation de l'ET de la quantité ingérée en réponse à la variation du poids initial ou de EX, et une augmentation de la variation du GMQ seulement avec une augmentation de la variation de EX. Ces auteurs ont également observé pour l'individu moyen une réponse de type linéaire-plateau du GMQ à l'augmentation de la surface disponible (de 0,40 à $0,80 \mathrm{~m}^{2}$ par porc) ou de la température (de 20 à $30^{\circ} \mathrm{C}$ ), alors que la réponse est de forme curvilinéaire-plateau pour la réponse moyenne d'une population de 500 animaux. Ceci illustre encore 
l'intérêt de prendre en compte la variabilité dans les modèles de croissance ainsi que la difficulté de cette prise en compte du fait des différentes sources de variabilité dont il faut tenir compte et du peu d'informations disponibles actuellement dans la littérature pour en estimer les effets, comme souligné par Wellock et al (2004).

\section{2 / Méthodes d'intégration de la variabilité dans les modèles}

L'intégration de la variabilité entre animaux dans les modèles de croissance du porc nécessite de connaître les valeurs moyennes et la variabilité, voire les valeurs individuelles des paramètres qui caractérisent un animal. Différentes méthodes ont été développées pour déterminer des paramètres tels que les masses protéiques et lipidiques à maturité et la vitesse de maturation. Ferguson et Gous (1993) proposent ainsi des abattages en série à différents stades de croissance pour analyser la composition chimique des animaux (protéines, lipides, matières minérales, eau) et ainsi calculer les paramètres recherchés par régression à partir de la fonction de Gompertz. Des mesures répétées de la composition corporelle peuvent être aussi réalisées in vivo pendant la croissance par méthode de mesure aux ultrasons ou tomographie (Schinckel et de Lange 1996), ce qui permet une estimation de la variabilité individuelle.

Des techniques alternatives de modélisation inversée ont également été développées pour obtenir les paramètres des modèles (Knap et al 2003, Doeschl-Wilson et al 2006). Une de ces techniques, dite par optimisation, consiste à ajuster itérativement les paramètres du modèle en comparant les observations avec les sorties du modèle (par exemple le GMQ) afin que les prévisions correspondent au mieux aux observations. L'obtention des paramètres et de leurs distributions permet d'inclure la variabilité dans les modèles pour étudier son impact sur les performances moyennes mais aussi sur leur variabilité. Dans l'exemple de Pomar et al (2003) ou dans d'autres études (Ferguson et al 1997, Knap 2000b, Wellock et al 2004), les simulations sont réalisées sur des populations générées à partir de la moyenne et de la variance d'un nombre limité de paramètres mais en considérant ceux-ci comme indépendants. Les covariances possibles sont donc ignorées, ce qui peut au final conduire à une surestimation de la variabilité simulée. L'introduction de la variabilité dans les modèles nécessite donc non seulement la connaissance des variances associées aux paramètres mais aussi des cova- riances entre paramètres (Ferguson et al 1997, Kyriazakis 1999), information qui n'est pas toujours disponible avec les méthodes décrites ci-dessus.

Schinckel et al (2003) et Strathe et al (2009, 2010) ont développé des modèles de croissance stochastiques en utilisant des fonctions non linéaires de modèle mixte, intégrant notamment une composante aléatoire. La détermination des paramètres de ces modèles s'appuie sur de la modélisation inversée et permet d'obtenir les valeurs individuelles des paramètres. Brossard et al (2009) ont également développé une approche basée sur la modélisation inversée en utilisant le modèle InraPorc ${ }^{\circledR}$. Celui-ci permet d'analyser les performances et l'utilisation des nutriments par l'animal et d'évaluer les conséquences d'un changement de stratégie alimentaire pour le porc en croissance et la truie (van Milgen et al 2008). Cet outil offre la possibilité d'estimer de façon dynamique les besoins nutritionnels. A partir de données individuelles de croissance et d'ingestion, Brossard et al (2009) déterminent pour chaque individu les paramètres qui permettent de décrire les dynamiques d'ingestion et de croissance. Dans InraPorc $\AA$, le dépôt de protéines est décrit par une fonction de Gompertz (van Milgen et al 2008). Ce dépôt fait évoluer la quantité de protéines corporelles qui, associée à celle des lipides corporels, permet de calculer le poids vif de l'animal et le gain de poids. Il existe ainsi une relation étroite entre dépôt protéique et gain de poids. De ce fait, la fonction de Gompertz a été utilisée dans cette étude pour décrire l'évolution du poids vif en fonction de l'âge. Une forme modifiée de la fonction de Gompertz permet de caractériser la croissance de chaque animal par trois paramètres : le poids vif à 65 jours d'âge $\left(\mathrm{PV}_{65 \mathrm{j}}\right)$, le paramètre de précocité $\mathrm{B}_{\text {Gompertz, et le GMQ sur la période } 65}$ jours - $110 \mathrm{~kg}$ de PV (GMQ $65 \mathrm{j}-110 \mathrm{~kg})$. L'âge de 65 jours et le poids final de $110 \mathrm{~kg}$ de PV ont été choisis car ils sont utilisés couramment en élevage comme limites de début et de fin de croissance. L'expression par rapport à ces limites permet de faciliter l'interprétation des paramètres. La Quantité d'aliment Ingéré à volonté (QI) est décrite dans cette étude par une fonction puissance du PV : QI = a x PVbconso. A partir de cette équation, deux paramètres sont utilisés pour caractériser l'ingéré de chaque animal : le paramètre de forme de la courbe $\left(\mathrm{b}_{\text {conso }}\right)$ et l'ingéré à $50 \mathrm{~kg}$ de PV $\left(\mathrm{QI}_{50}\right)$. Sans supposer des relations de cause à effet, ingestion et croissance sont corrélées, ce que reflètent les relations entre les paramètres du modèle obtenues pour une population de 192 porcs mâles castrés et femelles issus d'un croisement P76 x (Large White $x$ Landrace) et suivis en croissance entre 31 et $113 \mathrm{~kg}$ de PV (Brossard et al 2009). Il existe ainsi une corrélation forte entre $\mathrm{B}_{\mathrm{Gompertz}}$ et $\mathrm{b}_{\text {conso }},(-0,63 ; \mathrm{P}<0,01)$ indiquant qu'un animal avec une croissance précoce aura probablement aussi une augmentation précoce de son niveau d'ingestion. De même, la corrélation élevée entre $\mathrm{QI}_{50}$ et $\mathrm{GMQ}_{65 \mathrm{j}-110 \mathrm{~kg}}(0,66 ; \mathrm{P}<$ 0,01 ) indique qu'un animal ayant un niveau d'ingestion élevé à $50 \mathrm{~kg}\left(\mathrm{QI}_{50}\right)$ présentera probablement un GMQ élevé sur la période 65 jours - $110 \mathrm{~kg}$ de PV. Enfin, la corrélation quasi nulle et non significative entre $\mathrm{B}_{\mathrm{Gompert}}$ et $\mathrm{GMQ}_{65 \mathrm{j}-110 \mathrm{~kg}}(0,09 ; \mathrm{P} \stackrel{0,24)}{=}$ implique que deux animaux peuvent réaliser le même $\mathrm{GMQ}_{65 \mathrm{j}-110 \mathrm{~kg}}$ selon deux trajectoires de croissance différentes. La matrice de variance-covariance obtenue pour ces paramètres permet ensuite de générer d'autres populations de porcs, virtuelles cette fois, de caractéristiques moyennes identiques à la population d'origine mais de taille différente. Ces populations virtuelles sont utilisables en simulation pour étudier l'impact de différentes stratégies alimentaires, de différentes conduites (allotement, stratégie de départ à l'abattoir...). Cependant, les corrélations et la structure de variance-covariance observées entre paramètres dans une population donnée, ne sont à considérer que pour le type de porc étudié et dans des conditions d'élevage identiques à celles d'obtention des données.

La sensibilité des résultats de simulations aux variations des relations de variance-covariance doit être étudiée. Aussi, les relations de variance-covariance devront être caractérisées pour chaque jeu de données recueillies pour chaque croisement génétique et/ou condition d'élevage (par exemple, alimentation en groupe vs individuelle), afin de préciser si les relations observées sont tout ou partie généralisables entre types sexuels, croisements, etc. et quelle part de la variabilité peut être générée aléatoirement. L'obtention de données expérimentales ou issues de la pratique pour quantifier les paramètres et leurs interactions reste donc une étape incontournable dans les approches d'intégration de la variabilité individuelle dans les modèles. Enfin, la connaissance des valeurs des paramètres et des variances et covariances associées peuvent permettre de déterminer de façon plus réaliste le lien entre la variabilité de ces paramètres et celle des résultats de simulation, par exemple dans l'objectif d'établir des intervalles de confiance pour les valeurs prédites par les modèles. 
Figure 3. Evolution simulée du besoin en lysine digestible standardisée en fonction du poids vif au cours de la croissance chez une population de 192 porcs suivis entre $65 \mathrm{j}$ et $110 \mathrm{~kg}$ (valeurs moyennes et pour les $10 \%$ minimum et maximum de la population) (d'après Brossard et al 2009).

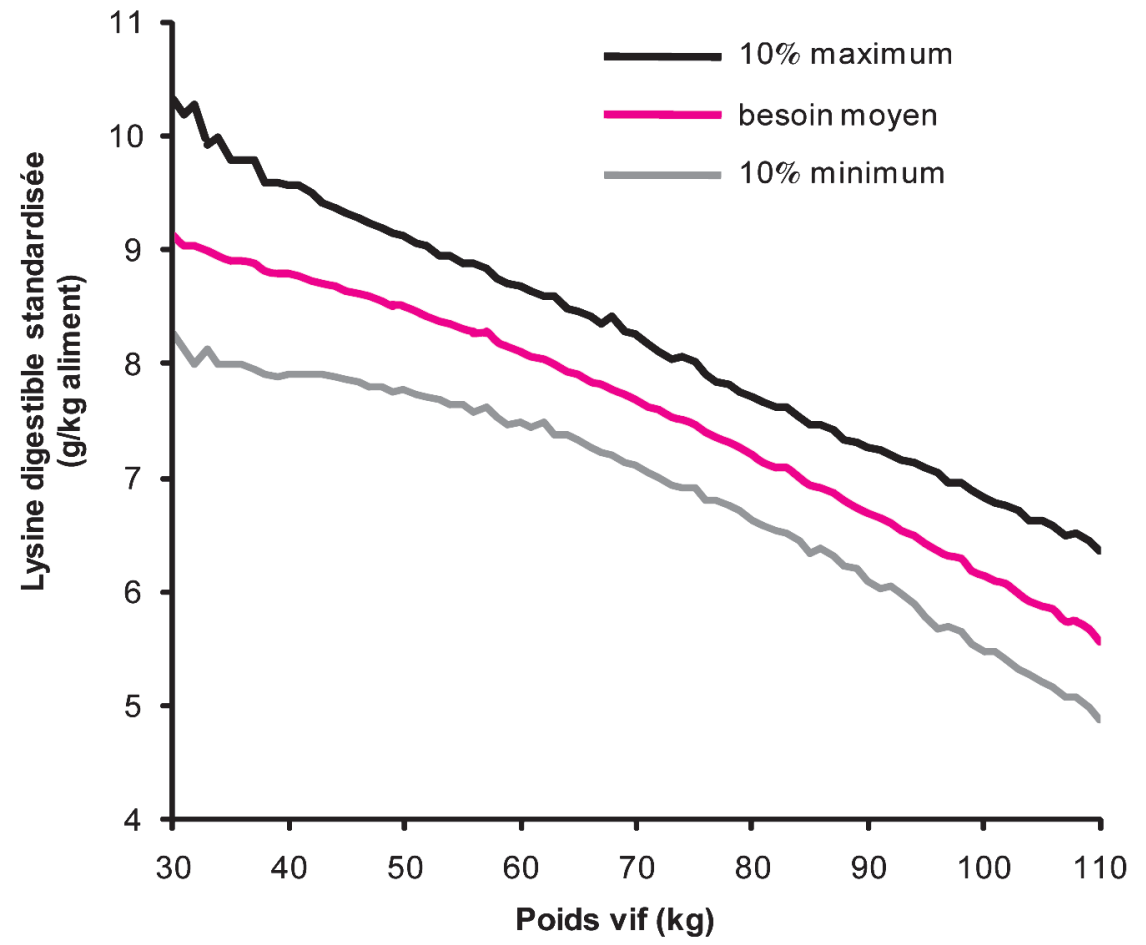

\section{3 / Analyse par modélisa- tion de la variabilité des besoins et de ses conséquen- ces sur l'évaluation des stratégies alimentaires}

\section{1 / Réponse à différentes stra- tégies alimentaires}

Les modèles mathématiques incluant la variabilité individuelle permettent de simuler les performances individuelles des animaux d'une population et d'estimer les besoins nutritionnels de la population (par exemple, Brossard et al 2009). Comme présenté ci-dessus, l'analyse à l'aide d'InraPorc $\AA$ des données individuelles de croissance et d'ingestion de 192 porcs a permis d'obtenir les valeurs des paramètres du modèle et par la suite les besoins en lysine digestible standardisée (LYSd) de chaque individu de la population (Brossard et al 2009). La figure 3 présente en fonction du poids vif l'évolution moyenne de ce besoin, exprimé en g par kg d'aliment et calculé à partir des besoins individuels. Les valeurs de besoin des $10 \%$ de porcs de la population présentant les besoins les plus bas et des $10 \%$ présentant les besoins les plus élevés sont également calculées. Ceci permet de constater la variabilité des besoins des animaux de la population: par exemple, le besoin moyen en LYSd à $31 \mathrm{~kg}$ de PV sera cou-
Brossard et al (2009) en simulant les performances de chaque individu. Des stratégies alimentaires à une, deux, trois ou 10 phases (correspondant à autant d'aliments différents utilisés pendant la croissance et changés à âge fixe) ont été appliquées en faisant varier l'apport en LYSd de 70 à $130 \%$ du besoin moyen de la population en début de phase pour chaque phase considérée. La stratégie à une phase correspond à une non prise en compte de l'évolution des besoins. Les stratégies à deux et trois phases sont couramment utilisées en production pour ajuster les apports au cours de la croissance. Enfin, la stratégie à 10 phases correspond à une application pratique possible pour un ajustement encore plus précis des apports au besoin. $\mathrm{La}$ gamme d'apports en LYSd a été choisie pour visualiser au mieux les variations d'apports nécessaires pour affecter les performances. Les résultats montrent que pour cette population, un apport en LYSd supérieur de $10 \%$ au besoin moyen de la population permet de satisfaire les besoins de plus de $90 \%$ des porcs de la population tout en maximisant le GMQ entre 65 jours d'âge et 110 $\mathrm{kg}$ de PV (figure 4) et minimisant l'indice de consommation, et ce quel que soit le nombre de phases appliquées sur la période de croissance.

Outre les performances moyennes, la vert par un apport de $9 \mathrm{~g} / \mathrm{kg}$ dans l'aliment, avec $80 \%$ de la population présentant un besoin compris entre 8,1 et $10,2 \mathrm{~g} / \mathrm{kg}$ d'aliment.

Les conséquences de la variabilité du besoin au cours de la croissance sur la réponse de la population à différents niveaux de lysine ont été étudiées par simulation des performances individuelles permet également de connaître la variabilité des performances des animaux. Ainsi, Brossard et al (2009) observent un accroissement du $\mathrm{CV}$ du GMQ avec l'augmentation du nombre de phases, ainsi qu'avec la diminution de l'apport en LYSd en deçà du besoin

Figure 4. Réponse simulée du gain moyen quotidien (GMQ, $\mathrm{kg} / \mathrm{j}$ ) (traits pleins) et $d u$ pourcentage d'animaux dont le besoin en lysine digestible standardisée (LYSd) est couvert (traits pointillés) en fonction de l'apport en LYSd (\% du besoin moyen de la population) chez une population de 192 porcs suivis entre $65 \mathrm{j}$ et $110 \mathrm{~kg}$ selon une stratégie alimentaire à 2 phases ou 10 phases (d'après Brossard et al 2009).

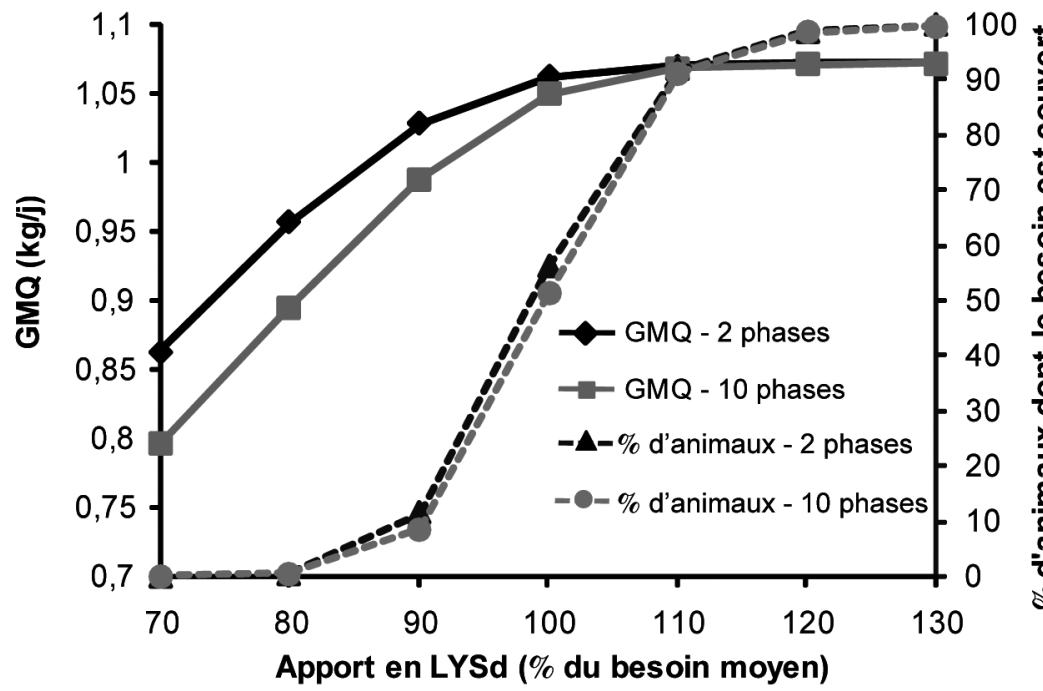

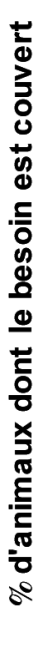


Figure 5. Evolution du Coefficient de Variation du Gain Moyen Quotidien (CV GMQ, $\%$ ) simulé en fonction de l'apport en lysine digestible standardisée (LYSd, \% du besoin moyen de la population) chez une population de 192 porcs suivis entre $65 j$ et $110 \mathrm{~kg}$ selon une stratégie alimentaire à 1, 2, 3 ou 10 phases (d'après Brossard et al 2009).

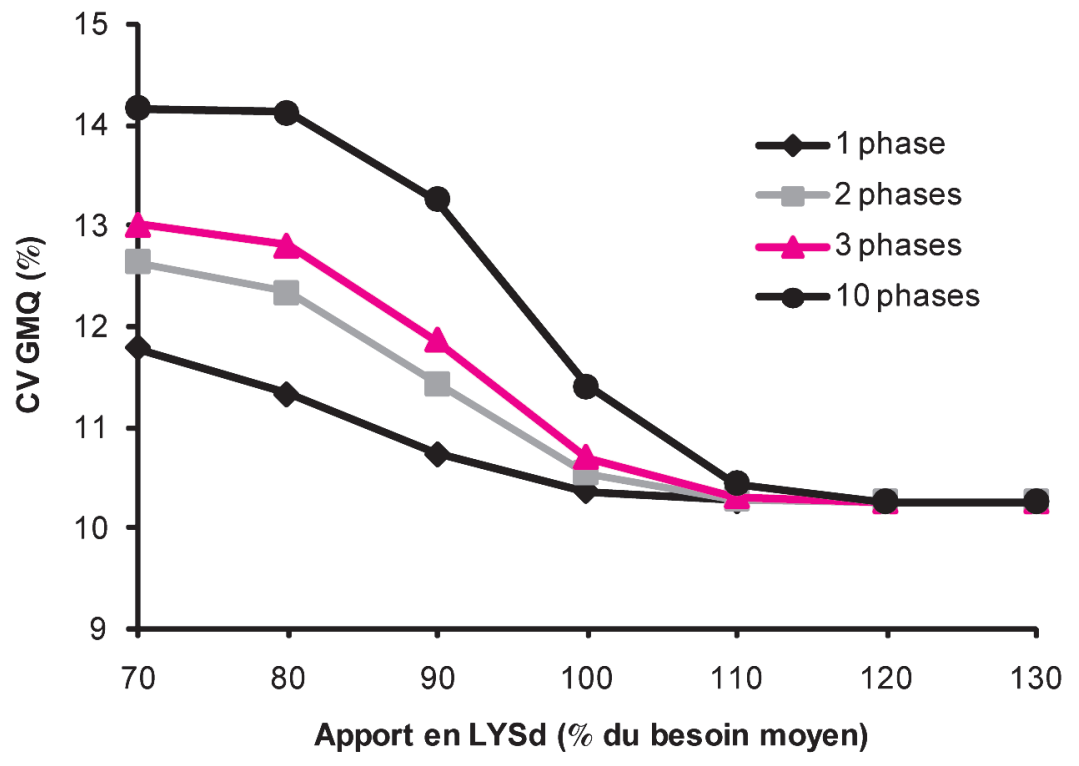

moyen (figure 5). En effet, avec la diminution des apports, spécialement pour les stratégies à plusieurs phases, le changement de phase à âge fixe implique que les animaux qui ont une performance plus faible que la moyenne, présentent un poids plus faible et donc un besoin plus élevé (en $\mathrm{g} / \mathrm{kg}$ d'aliment) que la moyenne au moment du changement d'aliment. Ils sont donc pénalisés par le changement d'aliment car leur besoin en lysine n'est pas couvert. Ceci contribue à amplifier les écarts de croissance et donc la variabilité. Pour des apports en LYSd supérieurs au besoin moyen, le CV est minimal quelle que soit la stratégie alimentaire et correspond alors à la variabilité naturelle de la population dans des conditions optimales.

Hauschild et al (2010b) ont également utilisé la modélisation des performances individuelles dans une comparaison entre les approches empiriques et factorielles de la détermination des besoins. Ces auteurs ont utilisé un modèle de croissance modifié à partir d'InraPorc ${ }^{\circledR}$ pour simuler les performances de 68 porcs en réponse à une augmentation du rapport entre la LYSd et l'Energie Nette (EN) ingérées et ainsi établir une loi de réponse par individu. Cela leur a permis d'estimer les besoins de ces animaux sur trois gammes de PV : de 26 à $53 \mathrm{~kg}$, de 54 à $81 \mathrm{~kg}$ et de 82 à $106 \mathrm{~kg}$. Dans leur étude, les valeurs de besoin en LYSd par unité d'EN ingérée sont distribuées normalement avec une moyenne de $0,96,0,73$ et $0,59 \mathrm{~g} / \mathrm{MJ}$ et un CV de $13,5,9,6$ et $9,6 \%$ respectivement dans la première, deuxième et troisième gamme de PV. Hauschild et al (2010b) ont également observé que le rapport minimal $\mathrm{LYSd} / \mathrm{EN}$ nécessaire pour optimiser les performances (qui est le besoin déterminé par la méthode empirique) est compris entre 100 et $112 \%$ du besoin moyen de la population selon le critère pris en compte (GMQ, indice de consommation) et la période de croissance.

Ces deux exemples confirment l'intérêt de considérer la réponse de la population et non plus celle de l'individu moyen. De plus, ils montrent qu'il est nécessaire de considérer à la fois la variabilité propre à chaque population à un moment donné et celle liée à l'évolution de l'âge des animaux. Ces deux composantes peuvent en effet conditionner l'ampleur des différences de réponse entre individu moyen et population.

\section{2 / Application de la modélisa- tion de la variabilité individuelle pour l'analyse multicritères des stratégies alimentaires}

Les exemples précédents illustrent que des performances de croissance maximales d'une population de porcs peuvent être obtenues en utilisant des aliments présentant par exemple un rapport LYSd/EN supérieur de quelques pourcents au besoin moyen. Ceci dépend également du nombre d'aliments utilisés pendant la croissance (Brossard et al 2009). En pratique, les teneurs en nutriments d'un aliment et le des contraintes de formulation, des nombre d'aliments utilisés dépendent contraintes propres à l'élevage (capacités de stockage et de distribution) ainsi que du prix et de la connaissance fine de la composition des matières premières. L'objectif est d'optimiser les performances de croissance mais également le paiement des carcasses (fonction du poids et du TMP) et le coût de production (coût alimentaire et utilisation des bâtiments) tout en respectant les normes environnementales. Le choix d'une stratégie alimentaire repose donc sur de multiples critères à appréhender simultanément. La réponse de la population étant différente de celle de l'individu moyen, il peut être intéressant d'intégrer la variabilité individuelle dans cette analyse notamment en ce qui concerne des critères comme les revenus liés à la vente des carcasses partiellement déterminés par la distribution des poids et du TMP (Quiniou et al 2012). La méthode de modélisation développée précédemment facilite cette analyse multicritère par rapport à l'expérimentation en permettant de modéliser l'évolution d'un nombre plus important de critères tout en affinant les résultats du fait de la prise en compte de la variabilité individuelle.

Quelques études illustrent 1'application de cette méthode. Morel et al (2008) ont étudié l'optimum économique de la teneur en Energie Digestible (ED) et du rapport LYSd/ED en fonction du génotype, de la taille de la population et de la variabilité dans la population. Des populations virtuelles de 1 à 625 porcs ont été créées aléatoirement en faisant varier quatre paramètres d'un modèle de croissance proche de celui décrit par Pomar et al (2003). La variation de ces paramètres autour de la moyenne a été illustrée par un CV de $0 \%$ (population d'un seul porc), $5,10 \mathrm{ou}$ $20 \%$ (populations de 5 à 625 porcs). Dans un premier cas, les paramètres étaient considérés comme indépendants et dans un second cas, les covariances entre ces paramètres étaient prises en compte dans la génération des populations. Les covariances utilisées étaient calculées à partir des corrélations observées dans des populations réelles et des variances déterminées par les CV appliqués et la moyenne des paramètres. Ces populations ont été créées pour trois types de porcs : maigre, normal et gras. Les régimes étaient changés chaque semaine. Les niveaux d'ED et du rapport LYSd/ED utilisés en formulation ont été déterminés par optimisation non linéaire basée sur un algorithme génétique (Morel et Wood 2005) sur la base des simulations des performances entre 20 et $85 \mathrm{~kg}$ de PV avec comme critère la maximisation de la marge brute finale. Cette marge brute a été calculée par porc par différence entre, d'une part, la valeur de la carcasse et, d'autre part, les 
Figure 6. Ratio Lysine digestible sur Energie Digestible (ED, $M J)$ pour maximiser la marge brute pour un porc seul (lignes noires) ou une population de 125 porcs avec un coefficient de variation des paramètres du modèle de $10 \%$ et une covariance entre ces paramètres (lignes rouges), pour des génotypes gras (losange), normal (carré) et maigre (rond) (d'après Morel et al 2008).

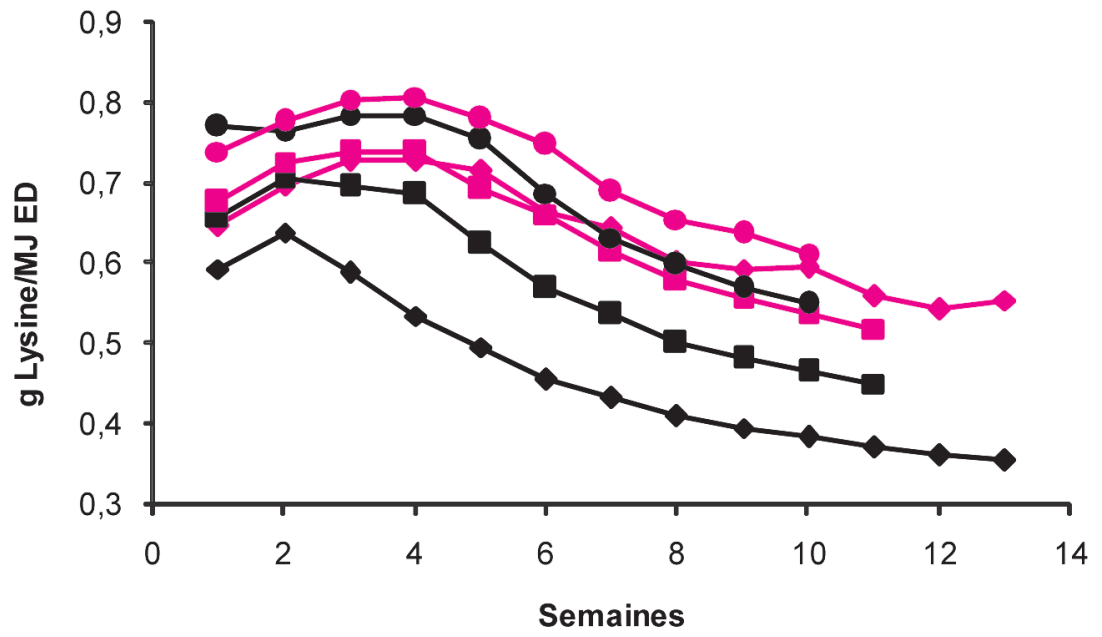

coûts alimentaires et le prix d'achat du porcelet. Les résultats montrent que c'est avec les porcs de type maigre que les meilleures marges brutes sont obtenues. La variabilité de cette marge augmente quand la variabilité de la population augmente mais diminue quand la taille de la population augmente. Quand la covariance entre paramètres est prise en compte, la comparaison des résultats entre la population d'un porc moyen et celle de 125 porcs indique que l'écart de rapport LYSd/ED optimal varie entre - $4 \%$ à $+50 \%$ selon le type de porc considéré (figure 6). L'écart entre les deux populations augmente au cours des 4 à 6 premières semaines de croissance puis reste stable. Les auteurs indiquent également que la prise en compte de la covariance entre paramètres fait augmenter les valeurs optimales du rapport LYSd/ED. Ceci reflète que la prise en compte de la variabilité individuelle, des relations entre paramètres mais aussi de la dynamique de ses effets sur les besoins, est importante.

Morel et Wood (2005) avaient déjà utilisé le modèle et la méthode décrits ci-dessus pour déterminer par optimisation les niveaux d'ED et du rapport LYSd/ED permettant de maximiser la marge brute mais également de minimiser l'excrétion d'azote. Les stratégies alimentaires comprenaient trois régimes formulés soit sur la base des spécifications disponibles commercialement (stratégies pratiques), soit à partir des valeurs optimisées d'ED et du rapport LYSd/ED (stratégies optimisées). Les performances étaient simulées pour 100 porcs avec un $\mathrm{CV}$ des paramètres du modèle de croissance de $10 \%$. Les auteurs observaient que les stratégies optimisées permettaient d'augmenter la le prix des aliments, mais également la teneur en protéines brutes dans les aliments, le contexte 2008-2009 permettant de formuler des aliments à moindre teneur en protéines et moins chers. Les quantités d'azote excrétées sont ainsi inférieures et le retour économique meilleur dans le contexte 2008-2009 (résultats non présentés). La stratégie alimentaire et le niveau du rapport LYSd/EN influencent également les résultats, en liaison avec le contexte économique. Les performances zootechniques maximales sont observées pour des apports en acides aminés supérieurs de 5 à $15 \%$ au besoin moyen (tableau 1). Ceci s'explique notamment par une amélioration du poids et de l'homogénéité des carcasses et du nombre d'animaux entrant dans la gamme optimale de TMP, comme montré par Quiniou et al (2007) par le même type d'analyse. La réduction du rapport LYSd/EN en dessous du besoin moyen induit une réduction des performances zootechniques et économiques plus marquée pour les stratégies d'alimentation en deux phases et d'alimentation multi-phase quotidienne avec une augmentation de l'excrétion d'azote pour la stratégie d'alimentation multi-phase quotidienne alors qu'elle diminue pour la stratégie à aliment unique. Avec l'augmentation du rapport LYSd/EN par rapport au besoin moyen, une amélioration des performances économiques est observée pour les stratégies d'alimentation en deux phases et multi-phase quotidienne avec un retour économique plus favorable pour la stratégie à deux phases par rapport aux autres stratégies. Dans ces conditions de rapport LYSd/EN, la stratégie d'alimentation multi-phase quotidienne permet un maintien des rejets azotés, les deux autres stratégies présentant des rejets supérieurs.

Les résultats ci-dessus montrent l'importance de la prise en compte simultanée de différents facteurs pour définir un optimum dans les apports nutritionnels. La variabilité au sein de la population, le contexte économique, l'importance donnée à la maximisation des résultats économiques et/ou à la minimisation des rejets sont en effet à considérer pour déterminer la valeur optimale par exemple du rapport LYSd/EN. L'évolution rapide du contexte économique, notamment du fait de la volatilité du prix des matières premières, et la variabilité entre groupes d'animaux impliquent également que ce type d'analyse indicative est à renouveler régulièrement pour s'adapter aux différentes situations rencontrées et est difficilement généralisable. Ceci amène également à s'interroger sur la notion de besoins nutritionnels. La définition physiologique de ceux-ci, comme étant 
Tableau 1. Effet de la stratégie alimentaire et de la teneur en lysine digestible relativement à l'énergie nette (LYSd/EN) dans les régimes sur l'indice de consommation, le retour financier (1) (contexte économique de septembre 2007) et l'excrétion d'azote pour une population virtuelle de 1000 porcs (d'après Brossard et al 2010).

\begin{tabular}{|c|c|c|c|c|c|c|c|c|}
\hline \multirow{2}{*}{ Variables } & \multirow{2}{*}{ Stratégie alimentaire } & \multicolumn{7}{|c|}{ Teneur en LYSd/EN (\% du besoin moyen) } \\
\cline { 3 - 9 } & & $\mathbf{8 5}$ & $\mathbf{9 0}$ & $\mathbf{9 5}$ & $\mathbf{1 0 0}$ & $\mathbf{1 0 5}$ & $\mathbf{1 1 0}$ & 115 \\
\hline \multirow{2}{*}{ Indice de } & Aliment unique & 2,75 & 2,70 & 2,67 & 2,66 & 2,66 & 2,65 & 2,65 \\
consommation & Deux phases & 2,88 & 2,79 & 2,72 & 2,69 & 2,67 & 2,66 & 2,65 \\
& Multi-phase quotidienne & 3,09 & 2,94 & 2,83 & 2,75 & 2,70 & 2,67 & 2,66 \\
\hline Retour & Aliment unique & $-3,8$ & $-1,8$ & $-0,6$ & 0,0 & 0,1 & 0,0 & $-0,4$ \\
financier & Deux phases & $-9,8$ & $-5,7$ & $-2,7$ & $-1,1$ & $-0,2$ & 0,2 & 0,2 \\
( $\Delta \in$ f/porc) & Multi-phase quotidienne & $-18,7$ & $-12,7$ & $-7,7$ & $-4,0$ & $-1,8$ & $-0,5$ & 0,0 \\
\hline Excrétion & Aliment unique & 3,14 & 3,27 & 3,44 & 3,61 & 3,84 & 3,88 & 3,88 \\
d'azote & Deux phases & 3,23 & 3,15 & 3,10 & 3,24 & 3,39 & 3,53 & 3,59 \\
(kg/porc) & Multi-phase quotidienne & 3,66 & 3,47 & 3,34 & 3,27 & 3,26 & 3,24 & 3,27 \\
\hline
\end{tabular}

(1) Différence relative au niveau LYSd/EN de $100 \%$ pour la stratégie à aliment unique.

la quantité de nutriments nécessaire pour maximiser un critère de production, doit tendre aujourd'hui à intégrer l'optimisation des résultats économiques et la réduction de l'impact environnemental, même si ce dernier point implique une réflexion à l'échelle de l'élevage mais aussi de la filière. Cette définition pourrait même s'étendre à des considérations sur le bien-être (satisfaction des besoins nutritionnels ou de l'appétit) et la santé des animaux ou sur l'impact sur le travail des éleveurs. L'intégration et la considération simultanée de ces différentes dimensions dans la notion de besoin sont difficilement réalisables sans le recours à la modélisation et l'incorporation de la variabilité individuelle dans les modèles. Les investissements en équipements de distribution d'aliment dont le coût peut varier selon les modalités d'alimentation retenues devraient également être considérés.

\section{4 / Vers une application en élevage}

L'analyse présentée ci-dessus de la variabilité des besoins et de ses conséquences technico-économiques incite à utiliser ces connaissances en élevage. Comme indiqué par Quiniou et al (2012), la variabilité des besoins entre animaux et son évolution au cours de la croissance est déjà prise en compte partiellement dans la pratique. Dans les élevages appliquant une stratégie alimentaire à deux aliments en engraissement (respectivement en périodes de croissance et de finition), des écarts plus ou moins importants de poids et donc de besoins nutritionnels sont observés entre animaux au changement d'aliment. La distribution de l'aliment de finition, moins concentré en acides ami- nés notamment, risque de pénaliser une partie des animaux. Il est alors conseillé aux éleveurs de constituer des cases homogènes en poids à l'entrée en engraissement et, quand les installations le permettent, d'adapter à chaque case selon le poids moyen le moment du passage à l'aliment de finition. Cet exemple fait partie des différentes stratégies disponibles pour améliorer les performances d'élevage tout en réduisant les rejets, à savoir une détermination précise des besoins nutritionnels, une formulation multicritère et un ajustement progressif des apports de nutriments aux besoins des porcs (Dourmad et al 2009, Pomar et al 2009a). Le développement

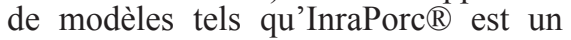
soutien à la prise en compte de la variabilité notamment dans la connaissance des besoins ou l'évaluation des stratégies alimentaires.

L'apport de la modélisation et la prise en compte de la variabilité individuelle amènent également à envisager le développement de nouvelles pratiques telles que l'alimentation de précision qui permet d'ajuster au mieux les apports alimentaires aux besoins nutritionnels. Dans ce cadre, la composition et la quantité de l'aliment sont ajustées quotidiennement selon les besoins estimés du groupe ou dans un objectif d'optimisation du revenu. Par rapport à une alimentation classique à deux ou trois aliments, ce type de stratégie alimentaire permet de réduire les rejets d'azote sans modifier les performances (Pomar et al 2007), ainsi que d'améliorer le retour économique (Niemi et al 2010). L'alimentation de précision peut également s'envisager par l'individualisation des apports alimentaires au sein du groupe. Pomar et al (2009b, 2010) ont ainsi montré par modélisation qu'une alimentation ajustée quotidiennement et individuellement aux besoins nutrition- nels de porcs en croissance permet de réduire les coûts alimentaires de $11 \%$ et l'excrétion d'azote et de phosphore de plus de $38 \%$ par rapport à une stratégie alimentaire à trois aliments appliqués au groupe dans son ensemble. L'alimentation de précision est encore en développement et nécessite de nouvelles méthodes de calcul pour son application en élevage, telles que des méthodes d'estimation journalière par modélisation des besoins nutritionnels individuels des porcs en croissance en fonction de leur parcours de croissance et de leur consommation (Hauschild et al 2010a). L'augmentation du nombre de phases ou l'ajustement quotidien de la composition de l'aliment peut toutefois se heurter à des difficultés techniques (nombre d'aliments différents à stocker, circuits de distribution) et entraîner des coûts supplémentaires. Ceci peut être résolu en mélangeant en proportion variable deux régimes formulés pour satisfaire les besoins en début et en fin de période de croissance (LetourneauMontminy et al 2005). Cette formulation, rendue complexe car relevant d'un problème non linéaire d'optimisation globale et nécessitant des algorithmes de résolution plus élaborés, a été en partie résolue par Letourneau-Montminy et al (2005). Ces auteurs ont appliqué un algorithme permettant de formuler les deux régimes simultanément et en calculant les proportions de mélange tout au long de la croissance. L'application en élevage de telles stratégies d'alimentation multi-phase quotidienne nécessite également des développements techniques. Par exemple, Feddes et al (2000) ont développé un système d'alimentation permettant d'automatiser le mélange de deux régimes dans des proportions établies et évoluant au cours du temps, et la distribution du mélange vers les nourrisseurs. 


\section{Conclusion}

La connaissance des besoins en acides aminés et de leur variabilité est nécessaire pour adapter l'alimentation des porcs en croissance afin d'optimiser les performances zootechniques et économiques tout en réduisant l'impact environnemental de cette production. En effet, la réponse de l'individu moyen représentatif d'un groupe ne reflète pas celle de la population. De plus, les résultats économiques d'un élevage dépendent en partie de la variabilité existant au sein de cet élevage. Les besoins individuels et leur variabilité peuvent être déterminés expérimentale- ment ou par modélisation. L'expérimentation présente toutefois des limites pour évaluer de manière directe la variabilité des besoins dans des contextes variés et sur un grand nombre d'animaux. C'est pourquoi différents modèles ont été développés et permettent de prendre en compte la variabilité des besoins selon différentes méthodes. Celles-ci ne permettent toutefois pas encore de prendre en compte toutes les sources de variabilité ni leurs effets combinés qui sont difficiles à estimer ou à modéliser du fait de leur non additivité. De plus, l'estimation des paramètres des modèles et de leur variabilité nécessitent un grand nombre de données zootechniques. Les liens entre modélisation et expérimentation restent donc étroits.
Les informations issues du phénotypage d'un nombre important d'animaux permettraient de caractériser et de quantifier les interactions entre caractères, pour mieux les intégrer aux modèles. D'un point de vue pratique, l'utilisation des modèles intégrant la variabilité individuelle permet d'analyser plus finement les effets des stratégies alimentaires sur des dimensions zootechniques, environnementales et économiques. Les informations obtenues permettent d'envisager des applications de ces concepts en élevage, telles que l'alimentation de précision. Ces applications nécessitent encore des développements tant au plan théorique que du matériel permettant leur mise en œuvre.

\section{Références}

Barea R., Brossard L., Le Floc'h N., Primot Y., Melchior D, van Milgen J., 2009. The standardized ileal digestible valine-to-lysine requirement ratio is at least seventy percent in postweaned piglets. J. Anim. Sci., 87, 935-947.

Bertolo R., Moehn S., Pencharz P., Ball R. 2005 . Estimate of the variability of the lysine requirement of growing pigs using the indicator amino acid oxidation technique. J. Anim. Sci., 83, 2535-2542.

Brossard L., Dourmad J.Y., Rivest J., van Milgen J., 2009. Modelling the variation in performance of a population of growing pig as affected by lysine supply and feeding strategy. Animal, 3, 1114-1123.

Brossard L., Quiniou N., Dourmad J., van Milgen J., 2010. A herd modelling approach to determine the economically and environmentally most interesting dietary amino acid level during the fattening period. In: Modelling nutrient digestion and utilisation in farm animals. Sauvant D., van Milgen J., Faverdin P., Friggens N. (Eds). Wageningen Academic Publishers, The Netherlands, 335-346.

Doeschl-Wilson A.B., Knap P.W., Kinghorn B.P., 2006. Evaluating animal genotypes through model inversion. In: Mechanistic modelling in pig and poultry production. Gous R., Morris T., Fisher C. (Eds). CABI Publishing, Wallingford, UK, 163-187.

Dourmad J.Y., Rigolot C., Jondreville C., 2009. Influence de la nutrition sur l'excrétion d'azote, de phosphore, de cuivre et de zinc des porcs, et sur les émissions d'ammoniac, de gaz à effet de serre et d'odeurs. In : Les nouveaux enjeux de la nutrition et de l'alimentation du porc. Montagne L., Dourmad J.Y. (Eds). Dossier, INRA Prod. Anim., 22, 41-48.

Feddes J.J.R., Ouellette C.A., Leonard J.J. 2000. A system for providing protein for pigs in intermediately sized grower/finisher barns. Can. Agric. Eng., 42, 209-213.

Ferguson N.S., Gous R.M., 1993. Evaluation of pig genotypes. 1. Theoretical aspects of measuring genetic parameters. Anim. Prod., $56,233-243$.

Ferguson N.S., Gous R.M., Emmans G.C., 1997. Predicting the effects of animal variation on growth and food intake in growing pigs using simulation modelling. Anim. Sci., 64 , 513-522.
Hauschild L., Lovatto P.A., Pomar J., Pomar C., 2010a. Estimation journalière des besoins nutritionnels individuels des porcs en croissance en fonction de leur parcours de croissance et de consommation. Journ. Rech. Porcine, 42, 99-106.

Hauschild L., Pomar C., Lovatto P.A. 2010b. Systematic comparison of the empirical and factorial methods used to estimate the nutrient requirements of growing pigs. Animal, 4, 714-723.

Heger J., Krizova L., Sustala M., Nitrayova S., Patras P., Hampel A., 2007. Assessment of statistical models describing individual and group response of pigs to threonine intake. J. Anim. Feed Sci., 16, 420-432.

Heger J., Krizova L., Sustala M., Nitrayova S., Patras P., Hampel D., 2008. Individual response of growing pigs to sulphur amino acid intake. J. Anim. Physiol. Anim. Nutr., 92, 18-28.

Heger J., Krizova L., Sustala M., Nitrayova S., Patras P., Hampel D., 2009. Individual response of growing pigs to lysine intake. J. Anim. Physiol. Anim. Nutr., 93, 538-546.

Henry Y., 1993. Affinement du concept de la protéine idéale chez le porc en croissance. INRA Prod. Anim., 6, 199-212.

Kim K.I., McMillan I., Bayley H.S., 1983. Determination of amino acid requirements of young pigs using an indicator amino acid. Brit. J. Nutr., 50, 369-382.

Knap P.W., 1995. Aspects of stochasticity: variation between animals. In: Modelling growth in the pig. Moughan P.J., Verstegen M.W.A Visser-Reyneveld M.I. (Eds). Wageningen Pers, The Netherlands, 165-172.

Knap P.W., 2000a. Variation in maintenance requirements of growing pigs in relation to body composition. A simulation study. Wageningen University, The Netherlands, 219p.

Knap P.W., 2000b. Stochastic simulation of growth in pigs: relations between body composition and maintenance requirements as mediated through protein turn-over and thermoregulation. Anim. Sci., 71, 11-30.

Knap P.W., Roehe R., Kolstad K., Pomar C. Luiting P., 2003. Characterisation of pig genotypes for growth modeling. J. Anim. Sci., 81, E187-E195.
Kyriazakis I., 1999. Future directions for models in pig biology. In: A quantitative biology of the pig. Kyriazakis I. (Ed). CABI Publishing, Wallingford, UK, 381-388.

Letourneau-Montminy M.P., Boucher C., Pomar C., Dubeau F., Dussault J.P., 2005. Impact de la méthode de formulation et du nombre de phases d'alimentation sur le coût d'alimentation et les rejets d'azote et de phosphore chez le porc charcutier. Journ. Rech Porcine, 37, 25-32.

Moehn S., Bertolo R.F.P., Pencharz P.B., Ball R.O., 2004. Indicator amino acid oxidation responds rapidly to changes in lysine or protein intake in growing and adult pigs. J. Nutr., 134, 836-841.

Moehn S., Shoveller A., Rademacher M., Ball R., 2008. An estimate of the methionine requirement and its variability in growing pigs using the indicator amino acid oxidation technique. J. Anim. Sci., 86, 364-369.

Morel P., Wood G., 2005. Optimisation of nutrient use to maximise profitability and minimise nitrogen excretion in pig meat production systems. Acta Hortic., 269-275.

Morel P., Wood G., Sirisatien D., 2008. Effect of genotype, population size and genotype variation on optimal diet determination for growing pigs. Acta Hortic., 287-292.

Moughan P., Smith W., Pearson G., 1987. Description and validation of a model simulating growth in the pig (20-90 kg liveweight). N.Z. J. Agric. Res., 30, 481-489.

Niemi J.K. Sevon-Aimonen M.L., Pietola K., Stalder K.J., 2010. The value of precision feeding technologies for grow-finish swine. Livest. Sci., 129, 13-23.

Noblet J., Quiniou N., 1999. Principaux facteurs de variation du besoin en acides aminés du porc en croissance. Techni-Porc, 22, 9-16.

NRC 1998. Nutrient requirement of swine, $10^{\text {th }}$ revised edition. National Academy Press, Washington, USA, 210p.

Pomar C., 2005. Nos modèles de simulation actuels sont-ils adéquats pour prédire la croissance corporelle et les besoins nutritionnels des porcs charcutiers ? Journ. Rech. Porcine, 37, 307-316. 
Pomar C., Kyriazakis I., Emmans G.C., Knap P.W., 2003. Modeling stochasticity: dealing with populations rather than individual pigs. J. Anim. Sci., 81, E178-E186.

Pomar C., Pomar J., Babot D., 2007. Effet d'une alimentation multi-phase quotidienne sur les performances zootechniques, la composition corporelle et les rejets d'azote et de phosphore du porc charcutier. Journ. Rech. Porcine, 39, 23-30.

Pomar C., Dubeau F., van Milgen J., 2009a. La détermination des besoins nutritionnels, la formulation multicritère et l'ajustement progressif des apports de nutriments aux besoins des porcs : des outils pour maîtriser les rejets d'azote et de phosphore. In : Les nouveaux enjeux de la nutrition et de l'alimentation du porc. Montagne L., Dourmad J.Y. (Eds). Dossier, INRA Prod. Anim., 22, 49-53.

Pomar C., Hauschild L., Zhang G.H., Pomar J., Lovatto P.A., 2009b. Applying precision feeding techniques in growing-finishing pig operations. Rev. Bras. Zootec., 38, 226-237.

Pomar C., Hauschild L., Zhang G., Pomar J., Lovatto P., 2010. Precision feeding can significantly reduce feeding cost and nutrient excretion in growing animals. In: Modelling nutrient digestion and utilisation in farm animals. Sauvant D., van Milgen J., Faverdin P., Friggens N. (Eds). Wageningen Academic Publishers, the Netherlands, 327-334.
Quiniou N., Massiot A., 2008. Alimenter à volonté ou rationner le porc charcutier. IFIP (Eds), Paris, France, 28p.

Quiniou N., Brossard L., Gaudré D., van Milgen J., Salaün Y., 2007. Optimum économique du niveau en acides aminés dans les aliments pour porcs charcutiers : impact du contexte de prix des matières premières et de la conduite d'élevage. TechniPorc, 30(2), 25-36.

Quiniou N., Brossard L, van Milgen J. Salaün Y., Quesnel H., Gondret F., Dourmad J.Y., 2012. La variabilité des performances animales en élevage porcin : description et implications pratiques. INRA Prod. Anim., 25, 5-16.

Schinckel A.P., Lange C.F.M., 1996. Characterization of growth parameters needed as inputs for pig growth models. J. Anim. Sci., 74, 2021-2036.

Schinckel A., Li N., Preckel P.V., Einstein M., Miller D., 2003. Development of a stochastic pig compositional growth model. Prof. Anim. Scientist, 19, 255-260.

Strathe A., Sorensen H., Danfaer A., 2009. A new mathematical model for combining growth and energy intake in animals: the case of the growing pig. J. Theor. Biol., 261, 165-175.

Strathe A.B., Danfaer A., Sorensen H., Kebreab E., 2010. A multilevel nonlinear mixed-effects approach to model growth in pigs. J. Anim. Sci., 88, 638-649. van Milgen J., Valancogne A., Dubois S., Dourmad J.Y., Sève B., Noblet J., 2008. InraPorc: A model and decision support tool for the nutrition of growing pigs. Anim. Feed Sci. Technol., 143, 387-405.

Warnants N., Oeckel M., Paepe M., 2003. Response of growing pigs to different levels of ileal standardised digestible lysine using diets balanced in threonine, methionine and tryptophan. Livest. Prod. Sci., 82, 201-209.

Wecke C., Liebert F., 2009. Lysine requirement studies in modern genotype barrows dependent on age, protein deposition and dietary lysine efficiency. J. Anim. Physiol. Anim. Nutr., 93, 295-304.

Wellock I.J., Emmans G.C., Kyriazakis I., 2003. Modelling the effects of thermal environment and dietary composition on pig performance: model logic and concepts. Anim. Sci., 77, 255-266.

Wellock I.J., Emmans G.C., Kyriazakis I., 2004. Modeling the effects of stressors on the performance of populations of pigs. J. Anim. Sci., 82, 2442-2450.

Whittemore C., Fawcett R., 1976. Theoretical aspects of a flexible model to simulate protein and lipid growth in pigs. Anim. Prod., 22, 87-96.

\section{Résumé}

Chez le porc en croissance, la connaissance des besoins nutritionnels, notamment en acides aminés, est nécessaire pour formuler des aliments permettant d'optimiser l'efficacité alimentaire et potentiellement de réduire l'impact environnemental de cette production. Cependant, tous les animaux ne présentent pas les mêmes besoins et donc pas les mêmes réponses à des apports équivalents, ce qui résulte de la variabilité du potentiel des animaux au sein d'un groupe. Cet article présente les outils disponibles pour évaluer la variabilité individuelle des besoins en acides aminés chez le porc en croissance. Nous montrons ensuite comment il est possible d'intégrer cette variabilité aux outils de modélisation afin d'optimiser les stratégies d'alimentation. Si les techniques expérimentales actuelles permettent une détermination individuelle des besoins, la représentativité des résultats obtenus sur la variabilité des besoins est limitée par le nombre restreint d'animaux étudiés. Tout en nécessitant un nombre important de données, l'intégration de la variabilité individuelle dans les modèles mathématiques de croissance du porc offre la possibilité d'analyser la variabilité des besoins de façon dynamique et sur des populations plus grandes. En utilisant ces modèles pour simuler des populations d'animaux et non plus un individu unique, des stratégies alimentaires adaptées aux populations considérées peuvent être définies pour optimiser leurs résultats zootechniques et économiques tout en minimisant l'impact environnemental. L'apport de la modélisation et la prise en compte de la variabilité individuelle amènent également à envisager le développement de nouvelles pratiques telles que l'alimentation de précision.

\section{Abstract}

\section{Accounting for individual variability in modelling the response of pigs to the nutrient supply}

Knowledge of amino acid requirements in growing pigs is required to formulate diets that optimize the feed efficiency and potentially limit the environmental impact of pig production. However, not all pigs have the same amino acid requirements, and thus the same responses to a defined nutrient supply, resulting from the variation in growth potential between pigs. The objective of this review was to discuss the tools available to assess the variation in amino acid requirements in growing pigs. We also demonstrate how the integration of variability in modelling tools is possible and allows optimizing feeding strategies. Current experimental techniques allow defining the requirement of individual animals, but the extrapolation of the results obtained is limited because few animals can be studied simultaneously. Although a sufficient number of data will be required, accounting for individual variability in pig growth models allows for a dynamic analysis of requirements of large populations. Using these models to simulate populations instead of a single average pig, feeding strategies can be defined that are adapted to the studied populations and optimize performance and economical results while reducing environmental impact. Accounting for individual variability in growth modelling is also essential to develop new technologies, such as precision feeding.

BROSSARD L., QUINIOU N., DOURMAD J.-Y., VAN MILGEN J., 2012. Prise en compte de la variabilité individuelle dans la modélisation de la réponse des porcs en croissance aux apports alimentaires. INRA Prod. Anim. 25, 17-28. 
